\title{
Morbilidade Neonatal e Cesariana Electiva em Recém-Nascidos de Termo
}

\author{
Neonatal Morbidity in Term Newborns Born by Elective Cesarean Section
}

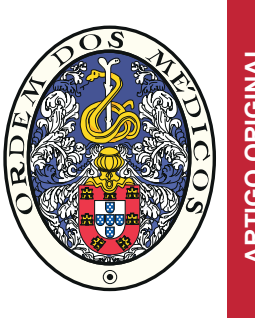

\author{
Maria Cristina RESENDE $\otimes^{1}$, Lea SANTOS ${ }^{2}$, Isabel SANTOS SILVA ${ }^{1}$ \\ Acta Med Port 2015 Sep-Oct;28(5):601-607
}

\section{RESUMO}

Introdução: A cesariana eletiva quando realizada antes das 39 semanas de idade gestacional associa-se a maior morbilidade neonatal e a maior risco de internamento em unidades de cuidados intensivos neonatais.

Objetivo: Avaliar a morbilidade neonatal em recém-nascidos de termo, nascidos por cesariana eletiva.

Material e Métodos: Estudo retrospetivo de todas as cesarianas eletivas realizadas com idade gestacional superior ou igual a 37 semanas numa maternidade de apoio perinatal diferenciado, nos últimos 11 anos (2003 - 2013). Foram excluídas as gestações de risco nomeadamente com rotura prematura de membranas, pré-eclampsia, diabetes mellitus mal controlada, isoimunização Rh, malformações congénitas e gestações múltiplas. Foi feita a comparação entre os grupos de recém-nascidos com idade gestacional inferior a 39 semanas e superior ou igual a 39 semanas.

Resultados: Da amostra de 3213 recém-nascidos, 45\% (1427) nasceram de cesariana eletiva antes das 39 semanas. Estes recémnascidos tiveram mais internamentos na Unidade de Cuidados Intensivos, odds ratio 2,4 [1,4 - 4,1] $p=0,001$, mais morbilidade respiratória, odds ratio de 2,4 [1,6 - 3,8] $p<0,001$, mais hiperbilirrubinémia com necessidade de fototerapia odds ratio 2,3 [1,5 - 3,7] $p<0,001$, mais hipoglicémia e/ou dificuldade alimentar odds ratio $1,6[1,2-2,4] p=0,006$ e mais internamentos com duração superior a cinco dias odds ratio $2,0[1,4-3] p<0,001$.

Discussão: Os recém-nascidos com idade gestacional inferior a 39 semanas tiveram maior morbilidade respiratória e metabólica e consequentemente tiveram maior número de dias de internamento.

Conclusão: Ao contrário do que está preconizado ainda existe na instituição um elevado número de cesarianas eletivas antes das 39 semanas. Devem ser programadas acções no sentido de sensibilizar os profissionais para este problema.

Palavras-chave: Cesariana; Nascimento a Termo; Procedimentos Cirúrgicos Eletivos; Recém-Nascido.

\section{ABSTRACT}

Introduction: International guidelines suggest that non-urgent planned deliveries be scheduled at or after 39 weeks. Despite this recommendation elective cesarean often occurs before 39 weeks. Some research has demonstrated that elective cesarean before 39 weeks poses a greater risk to the infants than at or after 39 weeks.

Objective: To evaluate neonatal morbidity in term newborns born by elective cesarean section.

Material and Methods: Retrospective study of all term elective cesarean sections (scheduled and without labor) performed in level III maternity, in the last 11 years (2003 - 2013). High risk pregnancies were excluded: twins, premature rupture of membranes, preeclampsia, poorly controlled diabetes mellitus, $\mathrm{Rh}$ isoimmunization and congenital malformations. Two groups of newborns with gestational age less than 39 weeks and equal or greater than 39 weeks gestational age were compared.

Results: In our sample, $45 \%$ of elective caesarean sections were performed before 39 weeks. Infants born before 39 weeks were more frequently admitted in neonatal intensive care, odds ratio $2.4[1.4-4.1] p=0.001$, had more respiratory morbidity, odds ratio 2.4 [1.6 $3.8] p<0.001$, more hyperbilirubinaemia odds ratio $2.3[1.5-3.7] p<0.001$, more hypoglycaemia and/or feeding difficulties odds ratio $1.6[1.2-2.4] p=0.006$, and longer admissions (more than five days), odds ratio $2.0[1.4-3] p<0.001$.

Discussion: As in other studies 'early term' had higher respiratory and metabolic morbidity and consequently had a longer hospital stay.

Conclusion: These findings support recommendations to delay elective cesarean delay until 39 weeks of gestation.

Keywords: Cesarean Section; Elective Surgical Procedures; Infant, Newborn; Term Birth.

\section{INTRODUÇÃO}

Define-se gravidez de termo a que tem a duração compreendida entre os 260 e os 294 dias $^{1}$ ou as 37 semanas e zero dias e as 41 semanas e seis dias. ${ }^{2}$

Tradicionalmente, o grupo de crianças nascidas neste intervalo de cinco semanas, é considerado como um grupo homogéneo de baixo risco. ${ }^{3-6}$ São considerados pré-termo os recém-nascidos (RN) que nascem antes das 37 semanas e zero dias e pós-termo as que nascem após as 42 semanas. Estas definições datam de 1970 e, já nessa al- tura alguns perinatalogistas preferiam adiar a definição de $\mathrm{RN}$ de termo para as 38 semanas e zero dias, pois argumentavam que os $\mathrm{RN}$ de 37 semanas eram ainda imaturos. Contudo, a definição de prematuridade da Organização Mundial de Saúde já estava enraizada em todos os profissionais de saúde ${ }^{2}$ pelo que se manteve as 37 semanas como limite inferior da definição de parto de termo.

Dado que a designação gravidez de termo tem implicações clínicas significativas no que diz respeito à gestão

\footnotetext{
1. Maternidade Bissaya Barreto. Centro Hospitalar e Universitário de Coimbra. Coimbra. Portugal.

2. Hospital Pediátrico de Coimbra. Centro Hospitalar e Universitário de Coimbra. Coimbra. Portugal.

$\triangle$ Autor correspondente: Cristina Resende. mcristina.resende@gmail.com

Recebido: 05 de Outubro de 2014 - Aceite: 22 de Julho de 2015 | Copyright $\odot$ Ordem dos Médicos 2015
} 
Tabela 1 - Nova classificação da duração da gravidez

\begin{tabular}{cc}
\hline Descrição & Idade gestacional \\
\hline Pré-termo & $<37$ semanas \\
Termo precoce & 37 semanas e 0 dias -38 semanas e 6 dias \\
Termo & 39 semanas e 0 dias -40 semanas e 6 dias \\
Termo tardio & 41 semanas 0 dias -41 semanas e 6 dias \\
Pós-termo & $>42$ semanas \\
\hline
\end{tabular}

de complicações na gravidez, de programação de parto electivo nomeadamente da cesariana eletiva, tem surgido necessidade duma reavaliação do conceito de gestação a termo. ${ }^{3-5} \mathrm{O}$ American College of Obstetricians and Gynecologists (ACOG) e a Society for Maternal-Fetal Medicine desaconselham o uso genérico de $\mathrm{RN}$ de termo e propõem uma nova classificação (Tabela 1). ${ }^{6}$

Os potenciais riscos do parto pré-termo e do parto pós-termo são sobejamente conhecidos e divulgados. Nas últimas décadas surgiram muitos estudos sobre os 'RN pré-termo tardios' ${ }^{7-8}$ erroneamente também denominados 'RN quase-termo'. Recentemente houve também uma chamada de atenção para os 'RN termo precoce' $\left(37^{\circ} S-38^{6} S\right)$ que quando comparados com RN com idade gestacional (IG) superior ou igual a 39 semanas apresentam maior mortalidade $^{9-12}$ e morbilidade neonatal. ${ }^{10-19}$

Os partos eletivos, incluindo a cesariana eletiva, têm aumentado por diferentes razões em todo o mundo, ${ }^{11-14}$ havendo um grande número de cesarianas eletivas por razões não médicas antes das 39 semanas. O ACOG publicou um boletim sobre esta temática, o Committee Opinion № 579 , que desaconselha a indução por razões não médicas antes das 39 semanas. ${ }^{20}$

$\mathrm{Na}$ última década tem havido uma preocupação crescente em informar as mães dos riscos de um parto induzido antes das 39 semanas. Duas grandes iniciativas como a Ohio Perinatal Quality Colaborative ${ }^{21} \mathrm{e}$ a Intermountain Health Care System in Utah ${ }^{22}$ já demostraram ser eficazes na redução da indução de partos por razões não médicas antes das 39 semanas. ${ }^{23-25} \mathrm{Em}$ contrapartida, com o aumento da idade gestacional há um risco aumentado de morbilidade neonatal por outros motivos nomeadamente o síndrome de aspiração meconial, macrossomia e consequentemente $\mathrm{o}$ traumatismo de parto ${ }^{26-28}$ e de morte fetal $\operatorname{tardia.}^{29,30}$

$\mathrm{Na}$ nossa maternidade apesar de não haver protocolo sobre esta temática, nos últimos anos têm havido uma preocupação crescente para diminuir a taxa de cesarianas eletivas antes das 39 semanas.

\section{OBJETIVO}

O objetivo deste estudo foi avaliar a morbilidade e mortalidade dos RN com IG inferior a 39 semanas nascidos por cesariana eletiva comparando-os com RN com idade igual ou superior a 39 semanas.

O objetivo secundário foi avaliar se nos últimos anos houve redução do número de cesarianas eletivas realizadas em idade gestacional inferior a 39 semanas.

\section{MATERIAL E MÉTODOS}

Estudo retrospetivo das crianças nascidas por cesariana eletiva realizada com IG entre as $37^{\circ} \mathrm{S}-41^{\circ} \mathrm{S}$, numa maternidade de nível de apoio perinatal diferenciado durante 11 anos (2003 a 2013). Foram excluídas situações de risco como gravidez múltipla, rotura prematura de membranas e patologias como pré-eclampsia, diabetes mellitus mal controlada, isoimunização $\mathrm{Rh}$, restrição de crescimento intrauterino e malformações congénitas.

Foi considerada cesariana eletiva a cesariana realizada, antes do início de trabalho de parto e antes da rotura de membranas. ${ }^{11}$

Calculámos a taxa de cesariana electiva e analisamos os motivos de realização da mesma ao longo dos 11 anos de estudo. Para avaliar a evolução das atitudes na programação da cesariana eletiva por razões não médicas, atendendo à ausência de um protocolo formal e para facilitar a análise dos dados, o estudo foi dividido em dois períodos temporais de igual dimensão, o primeiro de 1 de janeiro de 2003 a 30 de junho de 2008 e no segundo período de 1 de julho de 2008 a 31 de dezembro de 2013.

Avaliámos a morbilidade e mortalidade neonatal por IG e comparámos os resultados entre os RN com IG inferior a 39 semanas com os de IG igual ou superior a 39 semanas. Na morbilidade neonatal avaliámos a percentagem de RN com necessidade de internamento na Unidade de Cuidados Intensivos (UCIN), as complicações respiratórias (taquipneia transitória do RN, o síndrome de dificuldade respiratória (respiratory distress syndrome), necessidade de oxigenoterapia ou de suporte ventilatório), as complicações metabólicas (hipoglicémia definida como glicemia inferior a $50 \mathrm{mg} / \mathrm{dl}$, hiperbilirrubinémia com necessidade de fototerapia e a dificuldade alimentar), as complicações neurológicas (convulsões neonatais, encefalopatia hipóxico-isquémica, lesão do plexo braquial), a sépsis neonatal, o síndrome de aspiração meconial, o índice de APGAR inferior a 5 ao primeiro minuto e o internamento superior a cinco dias. Na nossa instituição habitualmente num parto por cesariana sem complicações os RN têm alta após as 72 
horas de vida, ou seja no quarto dia de vida, daí termos utilizado como ponto de corte o internamento superior a cinco dias, que traduz sempre alguma intercorrência pós-natal.

A análise estatística foi feita com o programa IBM SPSS Statistcs 20, considerando-se significância estatística se $p<0,05$. Foi feita a análise bivariada usando o teste $t$-Student - comparação entre médias (variáveis contínuas) e o teste Qui-quadrado/teste Fisher para comparação entre variáveis categóricas quando indicado pelas regras de Cochrane. Calcularam-se os odds ratio (OR) e os intervalos de confiança (IC).

\section{RESULTADOS}

Durante o período de estudo nasceram na nossa maternidade 29794 RN entre as 37 e as 41 semanas inclusive, Registaram-se 3123 cesarianas eletiva das quais 1427 (45\%) realizaram-se antes das 39 semanas de IG. A distribuição das cesarianas por IG está representada na Fig.1.

$\mathrm{Na}$ Tabela 2 podemos ver as características maternas e neonatais dos RN. As mães eram primíparas em $27 \%$ dos casos (851/3 123), tinham uma mediana de idade de 33 anos (mín. 14; máx 46). Os motivos da cesariana eletiva foram: cesariana anterior $n=1714(55 \%)$, a apresentação pélvica/transversa $n=888(28 \%)$, bacia geralmente apertada/suspeita de incompatibilidade feto-pélvica $n=176(6 \%)$, malformação uterina/ antecedentes de miomectomia $\mathrm{n}=$ $51(1,6 \%)$, suspeita de macrossomia fetal $n=37(1 \%)$ e doença materna impeditiva de parto vaginal $n=257(8 \%)$, das quais 34 eram portadoras do vírus de imunodeficiência humana.

Foram seguidas em médico particular $1648 / 3123$ das gravidezes (53\%) e em consulta hospitalar 35\% (1 096/3 123), foram seguidas conjuntamente no centro de saúde e

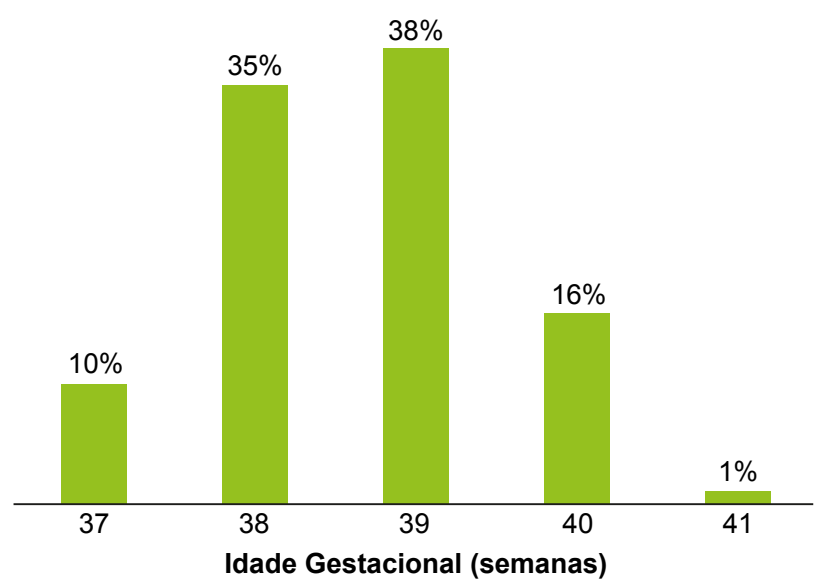

Figura 1 - Distribuição das cesarianas eletivas por idade gestacional

Tabela 2 - Distribuição das cesarianas eletivas nos 2 períodos consultas no hospital 310 (10\%). Eram do sexo masculino $49,7 \%$ (1555/3 123) dos RN; a mediana do peso ao nascer foi de 3300 gramas (mín. 2380 - máx. 5825 gramas); 6\% eram macrossomáticos, com peso superior a 4000 gramas $6 \%$ (196/3 123) dos RN e com peso superior a 4500 gramas $0,7 \%(23 / 3123)$. Apresentavam peso inferior a 2500 gramas, $2,3 \%$ dos $\mathrm{RN}$ (73/3 123).

A mediana do tempo de internamento foi de 4 dias, (min. 2 dias; máx. 32 dias).

$\mathrm{Na}$ Tabela 3 podemos ver a morbilidade neonatal nos RN por idade gestacional. Durante o período de estudo não houve mortalidade em RN de termo cujo parto foi a cesariana eletiva.

Necessitaram de reanimação neonatal 4,1\% (127/3 123) dos RN.

Foram internados na UCIN 2,2\% (63/3 123) dos RN. Às 37 semanas foram internados 4,3\% (14/323), às 38 semanas $2,5 \%(28 / 1104)$, às 39 semanas $1,1 \%(13 / 1170)$, às 40 semanas $1,6 \%(8 / 483)$, às 41 semanas não houve nenhum internamento na UCIN.

A distribuição das complicações respiratórias está representada na Fig. 2. Necessitaram de ventilação mecânica três, dos quais dois tinham IG inferior a 39 semanas.

Não houve nenhuma situação de convulsões, lesão do plexo braquial, encefalopatia hipóxico-isquémica ou sépsis. Dois RN, ambos de idade gestacional inferior a 39 semanas e com peso adequado à IG, apresentaram fracturas, uma do fémur e outra do úmero, devido a dificuldades na extracção fetal.

Avaliámos a evolução do número de cesarianas realizadas antes das 39 semanas em dois períodos. No primeiro período esta taxa foi de $58 \%$ e no segundo período de $42 \%$, $p<0,001$ (Tabela 4).

\section{DISCUSSÃO}

A nível mundial a cesariana eletiva tem vindo a aumentar. ${ }^{11,14}$ Existem razões médicas bem conhecidas para a indução do trabalho de parto antes das 39 semanas, onde se salienta a pré-eclampsia, a corioamnionite e a rotura prematura de membranas. Tem havido também um aumento das induções por razões não médicas, por conveniência ou vantagem quer para o médico quer para a futura mãe. Para além de parecer vantajoso por diminuir o risco de morte fetal, macrossomia, pré-eclampsia e evitar os incómodos do final da gravidez (edema, insónias e lombalgias) não há consciência da existência de riscos. A grande maioria dos 'RN termo precoces' não tem nenhuma complicação, nem requerem cuidados especiais; por outro lado as complicações são habitualmente de curta duração, sendo as complicações graves muito raras. ${ }^{31}$ Esta não consciencialização
$1 / 1 / 2003$ a $30 / 6 / 2008$
$1 / 7$ / 2008 a $31 / 12$ / 2013

p
$605(42 \%)$
$923(54 \%)$
$<39$ semanas
$822(58 \%)$
$773(46 \%)$

$$
<0,001
$$


Tabela 3 - Características maternas e neonatais

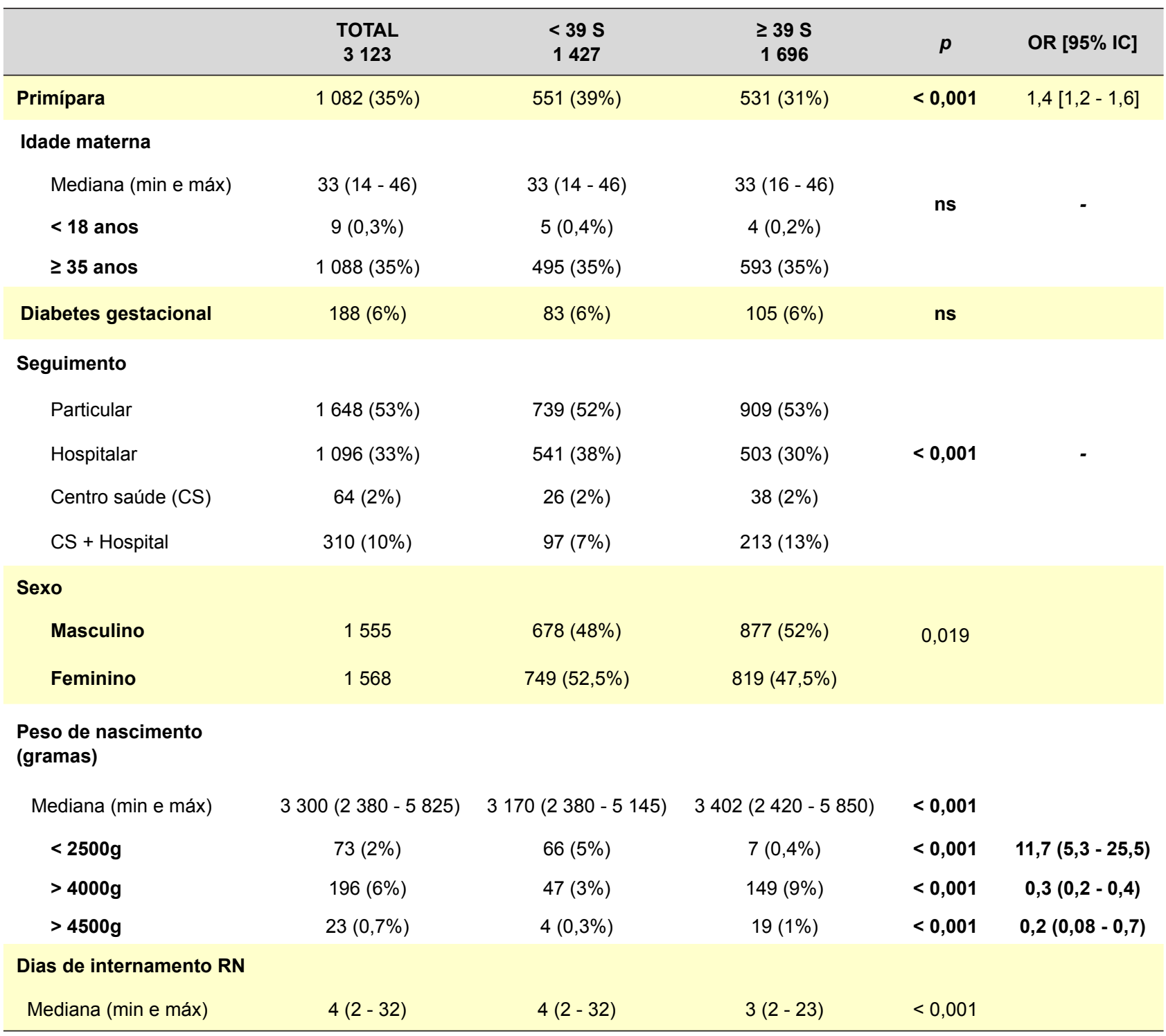

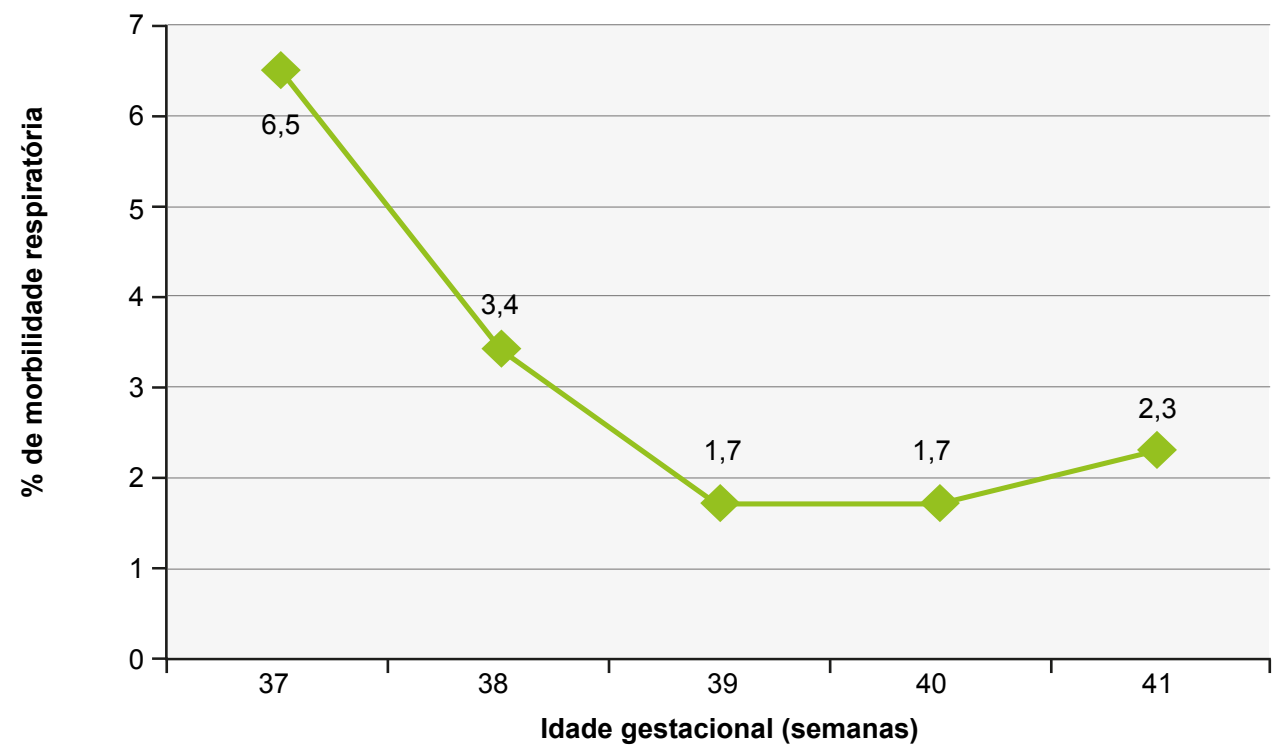

Figura 2 - Morbilidade respiratória por idade gestacional 
Tabela 4 - Morbilidade neonatal por idade gestacional

\begin{tabular}{|c|c|c|c|c|c|c|c|}
\hline $\begin{array}{l}\text { Idade gestacional } \\
\text { semanas }\end{array}$ & $\begin{array}{c}37 \\
n=323\end{array}$ & $\begin{array}{c}38 \\
n=1104\end{array}$ & \multicolumn{2}{|c|}{$\begin{array}{c}39 \\
n=1170\end{array}$} & $\begin{array}{c}40 \\
n=483\end{array}$ & $\begin{array}{c}41 \\
n=43\end{array}$ & $\begin{array}{l}\text { Total } \\
3123\end{array}$ \\
\hline Internamento UCIN & $\begin{array}{c}14(4,3 \%) \\
\text { OR - } 4(\mathbf{1 , 8}-\mathbf{8}, \mathbf{6})\end{array}$ & $\begin{array}{c}28(2,5 \%) \\
\text { OR - } 2,3(\mathbf{1}, \mathbf{1}-\mathbf{4 , 4})\end{array}$ & $\begin{array}{c}13 \\
(1,1 \%)\end{array}$ & & $\begin{array}{c}8(1,7 \%) \\
\text { OR - } 1,4(0,6-3,6)\end{array}$ & 0 & $\begin{array}{c}63 \\
(2 \%)\end{array}$ \\
\hline Sintomas respiratórios & $\begin{array}{c}21(6,5 \%) \\
\text { OR - } \mathbf{3 , 9}(\mathbf{2 , 1}-\mathbf{7 , 4})\end{array}$ & $\begin{array}{c}37(3,4 \%) \\
\text { OR - 1,9 }(2,1-3,4)\end{array}$ & $\begin{array}{c}20 \\
(1,7 \%)\end{array}$ & & $\begin{array}{c}8(1,7 \%) \\
\text { OR - } 0,9(0,4-2,2)\end{array}$ & $\begin{array}{c}1(2,3 \%) \\
\mathrm{OR}-1,3(0,1-10)\end{array}$ & $\begin{array}{c}87 \\
(2,8 \%)\end{array}$ \\
\hline $\begin{array}{l}\text { Hipoglicemia/ } \\
\text { dificuldade alimentar }\end{array}$ & $\begin{array}{c}13(4,0 \%) \\
\text { OR - } 0,7(0,4-1,4)\end{array}$ & $\begin{array}{c}58(5,3 \%) \\
\text { OR }-1,6(1,1-2,4)\end{array}$ & $\begin{array}{c}37 \\
(3,2 \%)\end{array}$ & $\frac{\pi}{0}$ & $\begin{array}{c}15(3,1 \%) \\
\text { OR - } 0,9(0,5-1,7)\end{array}$ & 0 & $\begin{array}{c}123 \\
(3,9 \%)\end{array}$ \\
\hline Hiperbilirrubinemia & $\begin{array}{c}19(5,9 \%) \\
\text { OR - } \mathbf{3 , 2}(\mathbf{1}, \mathbf{7}-\mathbf{6 , 1})\end{array}$ & $\begin{array}{c}37(3,4 \%) \\
0 R-1,8(1,1-3)\end{array}$ & $\begin{array}{c}22 \\
(1,9 \%)\end{array}$ & $\frac{\bar{d}}{\mathscr{\omega}}$ & $\begin{array}{c}6(1,2 \%) \\
\text { OR - } 0,6(0,2-1,6)\end{array}$ & $\begin{array}{c}1(2,3 \%) \\
0 R-1,2(0,1-9)\end{array}$ & $\begin{array}{c}85 \\
(2,7 \%)\end{array}$ \\
\hline Reanimação & $\begin{array}{c}21(6,5 \%) \\
\text { OR }-1,6(0,9-27\end{array}$ & $\begin{array}{c}34(3,1 \%) \\
\text { OR - } 0,7(0,4-1,1)\end{array}$ & $\begin{array}{c}48 \\
(4,1 \%)\end{array}$ & & $\begin{array}{c}22(4,6 \%) \\
\text { OR - } 1.1(0,6-1,8)\end{array}$ & $\begin{array}{c}2(4,7 \%) \\
0 R-1,1(0,2-4,8)\end{array}$ & $\begin{array}{c}127 \\
(4,1 \%)\end{array}$ \\
\hline Internamento $>5 d$ & $\begin{array}{c}26(8,0 \%) \\
\text { OR - 3,6 (2,1-6,3) }\end{array}$ & $\begin{array}{c}48(4,3 \%) \\
\text { OR - 1,9(1,1-3) }\end{array}$ & $\begin{array}{c}27 \\
(2,3 \%)\end{array}$ & & $\begin{array}{c}14(2,9 \%) \\
\text { OR }-1,2(0,6-2,4)\end{array}$ & $\begin{array}{c}3(7,0 \%) \\
\text { OR }-3,1(0,9-10)\end{array}$ & $\begin{array}{c}118 \\
(3,8 \%)\end{array}$ \\
\hline
\end{tabular}

do risco está bem patente num inquérito publicado em 2009 sobre a noção que as grávidas tinham em relação à IG em que achariam seguro nascer, e no qual $52 \%$ responderam que seria entre as 34 e 36 semanas. ${ }^{32}$ Num estudo semeIhante realizado em Portugal, 25,4\% responderam IG inferiores ou igual às 37 semanas. ${ }^{33}$

A grande maioria dos RN às $37-38$ semanas parece perfeitamente saudável e maduro. No nosso estudo $95,3 \%$ (1 361/1 427) dos RN com IG inferior a 39 semanas pesava mais de 2500 gramas. Mas sabe-se que estes RN são fisiologicamente imaturos e quando comparados com os RN de IG igual ou superior a 39 semanas têm maior necessidade de internamento na UCIN ${ }^{13-15,17,19}$ como também verificamos neste estudo.

A cesariana eletiva, mesmo às 39 semanas, têm um maior risco de morbilidade pois os RN são privados dos fenómenos hormonais e fisiológicos que ocorrem durante o trabalho de parto. Estes permitem uma adaptação pulmonar mediada pela libertação de catecolaminas que estimulam a reabsorção do líquido pulmonar fetal, diminuem a sua secreção e aumentam a produção de surfactante. Os 'RN termo precoce' têm a agravante de poderem apresentar algum grau de imaturidade pulmonar por défice de surfactante.

Em paralelo com outros estudos na literatura, os RN com IG inferior a 39 semanas tiveram maior morbilidade respiratória ${ }^{7,13-15,34-38}$ e metabólica ${ }^{13,15,16}$ e consequentemente tiveram maior número de dias de internamento. ${ }^{13-16}$

Verificámos que as cesarianas realizadas antes das 39 semanas foram mais frequentes em primíparas, justificamos o fato de terem sido realizadas em apresentação pélvica em primípara. Os RN com IG superior ou igual a 39 semanas eram em média mais pesados, com maior taxa de macrossómicos $(p<0,001)$, de acordo com a literatura. $14,28,36$

No nosso estudo a causa mais frequentes de cesariana

eletiva, foi a cesariana anterior $n=1714$. Uma cesariana prévia não constitui, por si só, uma indicação para nova cesariana em gestações subsequentes. Estudos nacionais e internacionais ${ }^{39,40}$ demonstraram que a maioria das grávidas submetidas a uma única cesariana pode ter um parto vaginal na gravidez seguinte, sem que exista um aumento significativo da mortalidade materna ou neonatal. $O$ fato deste estudo ser retrospetivo, limitou a avaliação dos motivos da realização da cesariana electiva. Contudo, nalguns casos em que o motivo principal foi a cesariana anterior verificamos que, associadamente, apresentavam outros diagnósticos secundários como: más condições de indução $n=438$, posição pélvica $n=148$, suspeita de macrossomia $n=128$, ou malformações uterinas/miomectomia anterior/ rotura uterina anterior $n=23$.

$\mathrm{Na}$ nossa maternidade tem havido nos últimos anos uma preocupação crescente para diminuir a taxa de cesarianas eletivas antes das 39 semanas, verificando-se uma diminuição com significado estatístico no segundo período do estudo. Avaliando apenas a morbilidade neonatal, a IG com menor risco parece ser as 39 semanas, ${ }^{14,28}$ pois após esta data existe um maior risco de morbilidade neonatal por outros motivos nomeadamente o síndrome de aspiração meconial, macrossomia e consequentemente o traumatismo de parto ${ }^{26-28}$ e de morte fetal tardia. ${ }^{29,30}$ Neste estudo em que se tratava na maioria dos casos de gravidezes de baixo risco não verificamos aumento deste tipo de morbilidade, tal como noutros estudos. Contudo em relação à mortalidade fetal ${ }^{22,29,30}$ existem outros factores a ter em consideração para além da IG, como a idade materna, índice de massa corporal pré-gravidez e comorbilidades como a diabetes e a hipertensão.

A idade gestacional ideal para realizar uma cesariana eletiva, é aquela que tem os melhores desenlaces para a mãe e para o RN, e não é necessariamente a mesma para todos. 


\section{CONCLUSÃO}

A data da programação de uma cesariana eletiva deve ser individualizada, no entanto os nossos resultados confirmam que os $\mathrm{RN}$ de cesarianas eletivas realizadas com idade gestacional inferior às 39 semanas têm um maior risco de admissão nas UCIN, de patologia respiratória, metabólica e maior duração de internamento.

\section{PROTECÇÃO DE PESSOAS E ANIMAIS}

A autora declara que os procedimentos seguidos estavam de acordo com os regulamentos estabelecidos pelos responsáveis da Comissão de Investigação Clínica e Ética e de acordo com a Declaração de Helsínquia da Associação Médica Mundial.

\section{CONFIDENCIALIDADE DOS DADOS}

A autora declara ter seguido os protocolos do seu centro de trabalho acerca da publicação de dados.

\section{CONFLITOS DE INTERESSE}

A autora declara que não houve conflitos de interesse na realização deste trabalho.

\section{FONTES DE FINANCIAMENTO}

Este trabalho não recebeu qualquer contribuição, subsídio ou bolsa.

\section{REFERÊNCIAS}

1. World Health Organization. ICD-10: International statistical classification of diseases and related health problems, $10^{\text {th }}$ revision. Volume 2. $2^{\text {nd }}$ ed. Geneva: WHO; 2004. [Consultado 2014 ago 28] Disponível em: http://www.who.int/classifications/icd/ICD-10_2nd_ ed_volume2.pdf.

2. European Association of Perinatal Medicine. Working party to discuss nomenclature based on gestational age and birth weight. Arch Dis Child. 1970;45:730.

3. Fleischman AR, Oinuma M, Clark SL. Rethinking the definition of term pregnancy. Obstet Gynecol. 2010;116:136-9.

4. Clark SL, Fleischman AR. Term pregnancy: time for a redefinition. Clin Perinatol. 2011;38:557-64.

5. Spong CY. Defining "term" pregnancy: recommendations from the Defining "Term" Pregnancy Workgroup. JAMA. 2013;309:2445-6.

6. American College of Obstetricians and Gynecologists. Definition of term pregnancy. Committee Opinion $\mathrm{N}^{\circ}$ 579. Obstet Gynecol. 2013;122:1139-40.

7. Engle WA, Kominiarek MA. Late preterm infants, early term infants, and timing of elective deliveries. Clin Perinatol. 2008;35:325-41.

8. Shapiro-Mendoza CK, Tomashek KM, Kotelchuck M, Barfield W, Nannini A, Weiss $\mathrm{J}$, et al. Effect of late-preterm birth and maternal medical conditions on newborn morbidity risk. Pediatrics. 2008;121:e223-32.

9. Reddy UM, Bettegowda VR, Dias T, Yamada-Kushnir T, Ko CW, Willinger M. Term pregnancy: a period of heterogeneous risk for infant mortality. Obstet Gynecol. 2011;117:1279-87.

10. Zhang X., Kramer MS. Variations in mortality and morbidity by gestational age among infants born at term. J Pediatr. 2009;154:35862.

11. De Luca R, Boulvain M, Irion O, Berner M, Pfister RE. Incidence of early neonatal mortality and morbidity after late-preterm and term cesarean delivery. Pediatrics. 2009;123:e1064-71.

12. Signore $\mathrm{C}$, Klebanoff M. Neonatal morbidity and mortality after elective cesarean delivery. Clin Perinatol. 2008;35:361-71.

13. Wilmink FA, Hukkelhoven CW, Lunshof $\mathrm{S}$, Mol BW, van der Post JA Papatsonis DN. Neonatal outcome following elective cesarean section beyond 37 weeks of gestation: a 7-year retrospective analysis of a national registry. Am J Obstet Gynecol. 2010;202:250.e1-8.

14. Ertugrul S, Gun I, Mungen E, Muhçu M, Kiliç S, Atay V. Evaluation of neonatal outcomes in elective repeat cesarean delivery at term according to weeks of gestation. J Obstet Gynaecol Res. 2013;39:10512.

15. Tita, AT, Landon MB, Spong CY, Lai Y, Leveno KJ, Varner MW, et al. Timing of elective repeat cesarean delivery at term and neonatal outcomes. N Engl J Med. 2009;360,111-20.

16. Sengupta S, Carrion V, Shelton J, Wynn RJ, Ryan RM, Singhal K, et al. Adverse neonatal outcomes associated with early-term birth. JAMA Pediatr. 2013;167:1053-9.

17. Hoffmire CA, Chess PR, Ben Saad T, Glantz JC. Elective delivery before 39 weeks: the risk of infant admission to the neonatal intensive care unit. Matern Child Health J. 2012;16:1053-62.

18. Clark SL, Miller DD, Belfort MA, Dildy GA, Frye DK, Meyers JA. Neonatal and maternal outcomes associated with elective term delivery. Am J Obstet Gynecol. 2009;200,156.e1-4.

19. Bailit, JL, Gregory KD, Reddy UM, Gonzalez-Quintero VH, Hibbard JU, Ramirez MM, et al. Maternal and neonatal outcomes by labor onset type and gestational age. Am J Obstet Gynecol. 2010;202:245.e1-12.

20. American College of Obstetricians and Gynecologists. Nonmedically indicated early-term deliveries. Committee Opinion No. 561. Obstet Gynecol. 2013;121:911-5.

21. The Ohio Perinatal Quality Collaborative Writing Committee. A statewide initiative to reduce inappropriate scheduled births at 36(0/7)38(6/7) weeks' gestation. Am J Obstet Gynecol. 2010;202:243.e1-8.

22. Oshiro BT, Henry E, Wilson J, Branch DW, Varner MW. Women and newborn clinical integration program. Decreasing elective deliveries before 39 weeks of gestation in an integrated health care system. Obstet Gynecol. 2009;113:804-11.

23. Fisch JM, English D, Pedaline S, Brooks K, Simhan HN. Labor induction process improvement: A patient quality-of-care initiative. Obstet Gynecol. 2009;113:797-803.

24. Ehrenthal DB, Hoffman MK, Jiang X, Ostrum G. Neonatal outcomes after implementation of guidelines limiting elective delivery before 39 weeks of gestation. Obstet Gynecol. 2011;118:1047-55.

25. Oshiro BT, Kowalewski L, Sappenfield W, Alter CC, Bettegowda VR, Russell R. et al. A multistate quality improvement program to decrease elective deliveries before 39 weeks of gestation. Obstet Gynecol. 2013;121:1025-31.

26. Caughey $A B$, Washington $A E$, Laros RK Jr. Neonatal complications of term pregnancy: rates by gestational age increase in a continuous not threshold, fashion. Am J Obstet Gynecol. 2005;192:185-90.

27. Caughey AB, Stotland NE, Washington AE, Escobar GJ. Maternal and obstetric complications of pregnancy are associated with increasing gestational age at term. Am J Obstet Gynecol. 2007;196:155.e1-6.

28. Cheng YW, Nicholson JM, Nakagawa S, Bruckner TA, Washington AE, Caughey $A B$. Perinatal outcomes in low-risk term pregnancies: do they differ by week of gestation? Am J Obstet Gynecol. 2008;199:370e1-7.

29. Page JM, Snowden JM, Cheng YW, Doss AE, Rosenstein MG, Caughey $A B$. The risk of stillbirth and infant death by each additional week of expectant management stratifies by maternal age. Am J Obstet Gynecol. 2013;209:375.e1-7.

30. Mandujano A, Waters TP, Myers SA. The risk of fetal death: current concepts of best gestational age for delivery. Am J Obstet Gynecol. 2013;208:207.e1-8.

31. Moore LE, Rayburn WF. Elective induction of labor. Clin Obstet Gynecol. 2006;49:698-704.

32. Goldenberg RL, McClure EM ,Bhattacharya A, Groat TA, Stahl PJ. Women's perceptions regarding the safety of births at various gestational ages. Obstet Gynecol. 2009;114:1254-8.

33. Lavaredas A, Milhinhos $\mathrm{C}$, Clode N. A percepção pela grávida da idade gestacional correspondente ao termo e segura para o nascimento do feto. Acta Obstet Ginecol Port. 2013;7:173-7.

34. Zanardo V, Simbi AK, Franzoi M, Solda G, Salvadori A, Trevisanuto D. Neonatal respiratory morbidity risk and mode of delivery at term: influence of timing of elective cesarean delivery. Acta Paediatr. 2004;93:643-7.

35. Van den Berg A, van Elburg RM, Van Geijn HP, Fetter WP. Neonatal respiratory morbidity following elective caesarean section in term 
infants: a 5-year retrospective study and a review of the literature. Eur J Obstet Gynecol Reprod Biol. 2001;98:9-13.

36. Ghartey K, Coletta J, Lizarraga L, Murphy E, Ananth CV, GyamfiBannerman C. Neonatal respiratory morbidity in the early term delivery. Am J Obstet Gynecol. 2012;207:292.e1-4.

37. Hansen AK, Wisborg K, Uldbjerg N, Henriksen TB. Risk of respiratory morbidity in term infants delivered by elective caesarean section: cohort study. BMJ. 2008;336:85-7.
38. Bates E, Rouse DJ, Mann ML, Chapman V, Carlo WA, Tita AT. Neonatal outcomes after demonstrated fetal lung maturity before 39 weeks of gestation Obstet Gynecol. 2010;116:1288-95.

39. American College of Obstetricians and Gynecologists. Vaginal birth after previous cesarean delivery. Practice Bulletin No.115. Obstet Gynecol. 2010;116:450-63.

40. Direção Geral de Saúde. Orientação n 003/2015 de 19/01/2015. Parto vaginal após cesariana electiva. Lisboa: DGS; 2015. 


\section{Morbilidade Neonatal e Cesariana Electiva em Recém-Nascidos de Termo}

Acta Med Port 2015:28:601-607

Publicado pela Acta Médica Portuguesa, a Revista Científica da Ordem dos Médicos

Av. Almirante Gago Coutinho, 151

1749-084 Lisboa, Portugal.

Tel: +351218428215

E-mail: submissao@actamedicaportuguesa.com

www.actamedicaportuguesa.com

ISSN:0870-399X | e-ISSN: 1646-0758

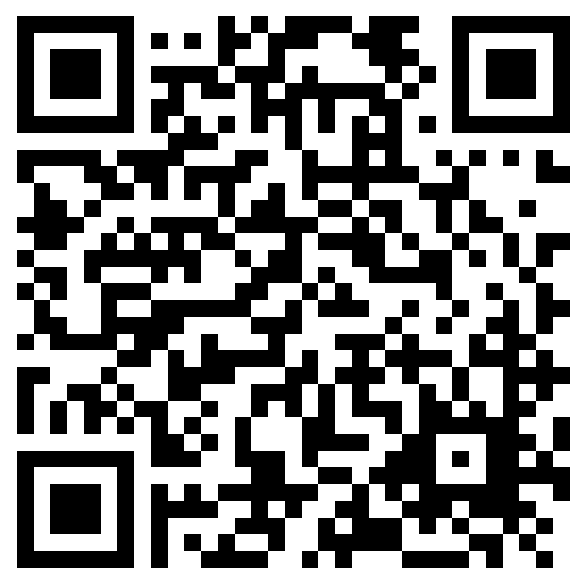

